\title{
Fuga lúdica: aspectos configurativos de la emergencia del jugar en la dinámica escolarizada contemporánea
}

\author{
Oscar Díaz Antillanca \\ Magíster en educación \\ Docente Universidad Austral de Chile \\ osfedian@gmail.com \\ Sergio Toro Arévalo \\ Doctor en ciencias de la educación mención didáctica \\ Docente Universidad Austral de Chile \\ sergio.toro@uach.cl
}

\section{Resumen}

Este artículo aborda el concepto de fuga lúdica y su relación con el contexto escolar, tensionando la experiencia del ser humano y su accionar desde la condición lúdica. A partir de la evidencia etnográfica de la investigación educacional "Complejidad y simpleza del juego" emerge la discusión de naturalizar el orden de los procesos escolares o escaparse a la potenciación de una condición biológica como lo es la lúdica. La reflexión sobre la fuga lúdica, trasciende el mismo juego y nos invita plantearnos ciertos interrogantes sobre el quehacer educativo y el viaje experiencial en la dinámica escolarizada contemporánea. El juego, la danza, la poesía, la música y el cuerpo o el lenguaje, la aritmética, la ciencia y el intelecto, entre muchos aspectos que convergen en la escuela, evidentemente no son antagonistas ni disyuntivos, sin embargo, se develan las dicotomías entre orden/desorden, serio/no serio, estudiar/jugar. Falsas dicotomías y trampas escolares que profundizan la liberación o la represión del elemento lúdico.

Palabras claves: fuga lúdica, juego, escuela, formación integral, orden escolar.

\section{Summary}

This article deals with the concept of playful leak and its relationship with the school context, stressing the experience of the human being and his action from the playful condition. From the ethnographic evidence of the educational research "Complexity and simplicity of the game" emerges the discussion of naturalizing the order of school processes or escaping to the strengthening of a biological condition as is the playful. The reflection on 
the playful leak transcends the same game and invites us to pose certain questions about the educational task and the experiential journey in the contemporary school dynamics. The game, dance, poetry, music and body or language, arithmetic, science and intellect, among many aspects that converge in the school, are obviously not antagonistic or disjunctive, however, dichotomies are unveiled between order/disorder, serious/not serious, study/play. False dichotomies and school traps that deepen the liberation or repression of the play element.

\section{Resumo}

Este artigo trata do conceito de fuga brincalhão e sua relação com o contexto escolar, enfatizando a experiência do ser humano e suas ações a partir da condição lúdica. A partir da evidência etnográfica da pesquisa educacional "Complexidade e simplicidade do jogo" surge a discussão de naturalizar a ordem dos processos escolares ou escapar da capacitação de uma condição biológica, como o jogo. A reflexão sobre o vôo lúdico, transcende o mesmo jogo e nos convida a colocar certas questões sobre a tarefa educativa e a jornada experiencial na dinâmica acadêmica contemporânea. O jogo, a dança, a poesia, a música e o corpo ou a linguagem, a aritmética, a ciência e o intelecto, entre muitos aspectos que convergem na escola, evidentemente não são antagonistas nem disjuntivos, no entanto, as dicotomias são reveladas entre ordem / desordem, grave / não grave, estudo / jogo. Falso dicotomias e armadilhas escolares que aprofundam a libertação ou a repressão do elemento lúdico.

Palabras claves: fuga brincalhão, jogo, escola, formação integral, ordem escolar.

\section{¿Fuga lúdica?}

Para fugarse de algo o de alguien, primero hay que encontrarse preso o en situación de encierro. Este concepto o reflexión en movimiento surge del trabajo etnográfico de la investigación "complejidad y simpleza del juego: análisis de las manifestaciones del juego en el contexto escolar y dimensión lúdica del educador de aula", perteneciente al proyecto fondecyt $\quad \mathrm{N}^{\circ} 1120761$ "conocimiento y comunidad escolar: procesos de interpretación de las dinámicas relacionales escolares en la educación como configuración cognitiva situada", cuyo objetivo central consistía en analizar la relación y tratamiento de la manifestación lúdica del juego en el terreno escolarizado. Dicha investigación, arrojó múltiples hallazgos y conceptos, tanto por las observaciones a los educadores como a los educandos en distintos niveles de enseñanza. Uno de los conceptos categorizados fue el de fuga lúdica. Este concepto hace alusión a la emergencia de algún tipo de manifestación del juego (ya sea de tipo recreativo o no) por parte de los estudiantes y/o docentes en un espacio determinado del contexto escolar. Generalmente esta emergencia del jugar, se presenta mayormente en los estudiantes que liberan la carga energética, mientras que los docentes la reprimen. La fuga lúdica, refleja un conflicto mucho más amplio, que es la negación de la condición lúdica del ser humano en el contexto escolar-total. Por tanto, el jugar, innegable al ser humano, cuando se contiene, sólo puede emerger de forma fugaz, impredecible y caótica; es decir como forma de escape. 
A continuación, se presentarán cinco fugas, que entremezclan conceptos reflexionados y algunas menciones a las observaciones con las cuales el concepto de fuga lúdica adquiere sentido.

\section{Primera Fuga: condición lúdica del ser humano}

Cuando un niño juega, lo único que le interesa en ese momento semióticotemporal único es el juego mismo. Más allá, de que existan grandes posibilidades de que ese juego esté propendiendo aprendizaje o pueda ser considerado como acceso a algún tipo de conocimiento específico, lo que suma relevancia es la acción voluntaria, libre, caótica, espontánea e incierta del jugar (Caillois, 1986). En cambio, cuando un niño estudia en la escuela, le interesan otras situaciones y eventos, simultáneamente a lo que está escuchando de su profesor. Lo que le está comunicando el compañero, compañera del lado, lo que sucede fuera del aula, alguna equivocación del profesor para transformarla en chiste, los dibujos que pudiera hacer en su cuaderno, alguna lluvia de papeles inminente o lo que está pensando realizar en el recreo. Muchas posibilidades que van edificando una intención lúdica constante.

Esta intención lúdica, la establecemos como un reconocimiento de la condición lúdica del ser humano, desde la infancia en adelante. El niño se reconoce y representa como un ser lúdico o en otras palabras como un homo ludens (Huizinga, 2000). Sin embargo, no tiene que ver solamente con el hombre del juego o el hombre que juega. Tiene que ver con la forma en que el ser humano observa-conoce-experiencia el mundo (Agamben, 2011), a través de dinámicas e interacciones fluctuantes de carácter sinérgico, consideradas lúdicas.

El sentido de lo lúdico entra en juego. El jugar por jugar es válido. Pero el niño nunca está jugando por jugar. Para ellos el juego puede tornarse bastante serio, por lo cual tiene un sentido adquirido antes, durante y después del juego. El niño puede ver esas categorías, niveles, habilidades y/o aspectos que está aprendiendo y co-aprendiendo en el ambiente cultural en la cual está inmerso, formando una reflexión del jugar bastante interesante.

Desde el espacio pre-escolarizado, el niño viene aprendiendo el mundo de forma libre, espontánea y natural. Sin embargo, al llegar al aula ese espacio libre, posee otras condiciones. Estas condiciones van entrampando la aceptación naturalizada de la condición lúdica. Pareciera ser que, al momento de ingresar a la escuela, el tiempo de jugar se acabó, porque hay dimensiones consideradas más relevantes para potenciar (Calvo, 2007). Ni hablar de un desarrollo holístico en el aula, cuando todo está ordenado y apuntando a la cabeza del niño, olvidándose conscientemente del resto del cuerpo (Naranjo, 2010). En las observaciones y entrevistas a los educadores se evidenció una similitud en la forma de entregar el conocimiento a los niños, niñas y jóvenes. La estandarización y aplicación de los programas escolares se aplicaba correctamente según pautas preestablecidas (Illich, 1997). Sin embargo, los mismos educadores manifestaron que no pueden enseñar a través de actividades lúdicas, porque para el contexto escolar significa desorden. A su vez, tampoco pueden ser el profesor que quieren ser, ya que son el profesor que deben ser. Entonces, se reconocen como seres lúdicos, pero juegan, se 
divierten, se recrean, son más libres en su casa porque en la escuela las interacciones que se necesitan son distintas. Mientras, que los educandos de vez en cuando liberan sus juegos reprimidos, y es ahí, donde se arma el conflicto. Podemos indicar que el mundo casa y el mundo escuela se contrastan más que acercarse a una armonía en la praxis.

\section{Segunda fuga: emergencia del juego}

No es una exageración que la mayoría de los seres humanos hemos jugado y aprendido cosas simples o complejas a través del juego. Si el niño ha estado jugando durante sus primeros años, es lógico que lo quiera seguir haciendo, inclusive, cuando está en la escuela "estudiando". Las redes sistémicas de la escuela inhiben la posibilidad de movimiento incierto y de descubrimiento placentero del niño (Buytendijk, 1935). Existe todo un sistema de vigilancia y control, para que las reglas se cumplan, para que los contenidos se apliquen y para que los que las rompen, reciban castigo (Illich, 1977). Es por esto, que cuando suena el timbre después de cada clase de 90 minutos, se observa que los educandos salen al momento corto de ocio y tiempo libre, denominado recreo, como verdaderos reclusos privados de su libertad. Y si bien el recreo es un momento de más caos en el contexto escolar, está permitido, porque en caso de que el orden se altere demasiado, los inspectores pondrán en su lugar a los rebeldes. En definitiva, la emergencia del juego siempre está siendo observada, porque si aparece una fuga lúdica, debe reprimirse de inmediato. Pero, a qué nos referimos concretamente con ¿Emergencia del juego? Para comprender esta característica tenemos que volver al principio. La lúdica, ha sido estudiada desde diferentes disciplinas. Por ende, existen variadas clasificaciones sugeridas. Puede ser entendida como "medio para", "instrumento" para acceder al conocimiento, cuyos fines pueden o no ser pedagógicos. Desde una perspectiva pedagógica (escuela), puede ser catalogada como una actividad que permite propender aprendizaje de manera más dinámica y dialógica (posibilidad didáctica-formativa), en cambio desde una perspectiva socio-cultural, no sólo es un instrumento, sino una manifestación humana, una expresión de la cultura. Ya en el campo de las ciencias humanas, existen algunas confusiones. La lúdica como sinónimo de juego, analizado de forma instrumental, que también podría ser productivo. En cambio, desde una perspectiva más abierta, el juego es sólo una las tantas manifestaciones de la lúdica, por excelencia, considerando el elemento lúdico, como una condición biocultural, de la naturaleza humana, que debe desarrollarse de forma armónica para nuestra salud física y cognitiva, simplemente para satisfacer una necesidad lúdica vital (urgenciaemergencia de llevar a cabo acciones de forma libre, espontáneas, caóticas, inciertas e inéditas), a través de las actividad lúdica (acción para liberar voluntariamente el impulso generado por la necesidad, a través de movimientos [realidad] o pensamientos [imaginación]) y por último, el placer lúdico (el disfrute de la experiencia inmediata, o la consecuencia lograda por la necesidad satisfecha) Jugar. Nos quedamos netamente con este impulso impredecible y totalmente libre.

\section{Tercera fuga: conflicto lúdico en el orden escolar y falsa dicotomía}

El conocimiento universal que poseemos sobre las formas, redes, mecánicas y 
relaciones en la complejidad del contexto escolarizado, nos permite coincidir en algunos criterios unificados que resaltan este orbe y que entran en conflicto con la condición lúdica:

1) Orden

2) Planificación

3) Asimetría

4) Estandarización

5) Homogeneización

6) Medición

7) Fragmentación

8) Competencia

El orden en el contexto escolar es la idea naturalizada de entender la quietud de los niños, como sinónimo de escenario ideal para el aprendizaje. Se orienta el orden netamente a la disciplina. Disciplina que no permite movimiento, creatividad, pensamiento divergente o ningún acceso alternativo del niño al conocimiento, que no sea el planificado escolarmente. $\mathrm{La}$ planificación, dónde se encuentran los objetivos anuales que se traza la institución, las metas académicas, las actividades conmemorativas, salidas pedagógicas, festivos, etc. Todo está diseñado al vacío, con mucha anticipación. La asimetría, entendida como la jerarquía en las relaciones, que prevalece sobre relaciones cercanas y comunitario-sociales, señalando en cada instante el título autoritario, sobre afinidades e intereses comunes (Calvo, 2007). La estandarización, pilar fundamental intencionado del contexto escolar, que pregona el conocimiento universal, enseñado de igual forma para diferentes seres humanos, con ritmos $\mathrm{y}$ momentos de aprendizaje distintos. Es decir, diferentes inteligencias aprenden sólo una inteligencia que se supone es la relevante. $\mathrm{Ni}$ hablar del concepto de ecología de mentes, que en el contexto escolar es una utopía. Se fabrican las mentes de los niños en serie, como un producto, ajustándose al estándar que, en este caso, es una estándar neo-liberal (Luna, 2008). La homogeneización de los niños, reprimiendo todos sus elementos que los hacen ser distintos en un cuerpo uniforme, carente de las propiedades innatas que podrían potenciarse. La medición, como garantía de una idea de educación poco definida, superflua, que responde a un paradigma estático. La misma prueba para medir contenidos, dejando de lado habilidades y posibilidades ingeniosas de resolución (Illich, 1997). La fragmentación, que atraviesa los cimientos biológicos e intelectuales de los niños, logrando parcialidades del conocimiento, en lugar de uno que sea holístico, situado y encarnado; la competencia que suprime el trabajo colaborativo, como simulación de la vida post-escolar, donde el trabajo se nutre por la eliminación del resto de postulantes. Los niños son divididos en personas académicas y no académicas en el contexto escolar y posteriormente en personas laborales y no laborales en el contexto social.

Estos criterios, provocan un conflicto artificial pero evidente con el niño, y no sólo con él, sino con su ritmo de aprendizaje, su manera de aprender, sus distinciones, en definitiva, con su forma de acceder al conocimiento del mundo, de la inocencia total de descubrirse a través de las emociones (Maturana, 2003). Simultáneamente, una falsa dicotomía entre lo serio y lo no serio, reflejada en el estudiar como exclusivamente serio y el jugar como extremadamente no serio.

\section{Cuarta fuga: conciencia del engaño lúdico y doble control}

Al pasar una buena cantidad de tiempo en la escuela, ya posees total sabiduría del 
funcionamiento correcto de la dinámica escolarizada. Adquieres una especie de conciencia del engaño lúdico, internalizando la dicotomía artificial de la acción de estudiar como algo serio y la acción de jugar como algo no serio ni productivo. El tema es que, al pasar el tiempo, el ser humano escolarizado se pierde en la naturalización de suprimir el elemento lúdico, aplicando un doble control. Por un lado, el contexto escolar contiene la condición lúdica como agente externo y, por otro lado, el yo mismo, aplica la contención interna: doble control. La regulación y autorregulación, influye directamente en la liberación del impulso vital lúdico y en la posibilidad de nuevas interacciones formativas. Sin el desarrollo adecuado de la condición lúdica se sufren consecuencias biológicas, epistémicas y emocionales (Paymal, 2010), que a su vez derivan en un doble control prejudicial y recursivo. $\mathrm{La}$ necesidad de la fuga lúdica y del jugar para configurarnos como seres humanos (Schiller, 1990), se torna urgente, pero ¿Es suficiente? La incompatibilidad de los jugares en el contexto escolar, es decir, el juego del docente versus el juego del discente, nos indica que no. No es suficiente habitar en la dinámica escolarizada sólo fugándose a lo lúdico, porque limita la experiencia misma de saberse lúdico y otorgarle el sentido necesario para adquirir la reflexión y conciencia de la configuración de la emergencia del jugar (Maturana, 2003).

\section{Quinta fuga: liberación completada o fuga lúdica reprimida}

El proceso educativo escolarizado se bifurca exclusivamente en la disciplinaorden (exigida por los educadores) y la indisciplina -desorden (propuesta por los educandos). Por lo mismo, las fugas lúdicas, que la mayoría de las veces emergen desde los aprendientes, son rápidamente suprimidas. A pesar de esto, los docentes demuestran elevados grados de crítica sobre sus propias prácticas, entonces ¿Es un problema sistémico? Al parecer el contexto escolar mismo se vuelve ajeno a los elementos vitales que nos consolidan como ser que aprende, que busca, que erra y que juega. Sin embargo, en la simulación de aprender los contenidos escolares en el territorio escolar (Calvo, 2010) somos nosotros mismos los que construimos la escuela y la esencia de esa escuela día tras día. Las personas son el sistema, y en un análisis lógico, si el sistema está en crisis, nosotros también lo estamos (Naranjo, 2007). Como seres humanos en constante crisis, se establecen dos opciones evidentes con nuestra condición lúdica en el contexto escolar: a) aceptar la innegabilidad de lo lúdico y ejercer una liberación consciente, de que a pesar de la marginación escolar hacia lo lúdico, debemos-podemos explorar el mundo a través de las dinámicas caóticas, complejas, espontáneas, libres e inciertas b) reprimir la intención de la fuga lúdica y aceptar no habitar por el desarrollo de la condición lúdica, asumiendo los costes antes mencionados. La liberación completada o la fuga lúdica reprimida dependerá de una serie de factores aleatorios e impredecibles, donde la última palabra siempre la tendrá el jugador.

\section{Conclusiones}

La relevancia de la lúdica y sus expresiones por excelencia como el juego, la danza, la música, la poesía, el arte, la fiesta y el ritual, dan cuenta que el ser humano experiencia el mundo a través de interacciones dinámicas libres y autorreguladas que influyen e impactan en su desarrollo físico, emocional, 
cognitivo, social, político y espiritual. Existe una discriminación, en primer lugar, de carácter escolar, entendida como persona académica o no-académica, $\mathrm{y}$, en segundo lugar, de carácter laboral, como persona productiva o no productiva. Evidente discriminación es artificial y engañosa. Una trampa que se ha naturalizado en las bases de la cultura, cuyas competencias neo-liberales adquieren mayor sentido y profundidad, en desmedro (olvido, ceguera, control, negación consciente) de otras dimensiones del ser a potenciar, como la condición lúdica, innegable al ser humano para descubrir, conocer, reconocer, aprender, aprehender, recrear el mundo y que en el contexto escolar se presenta como fuga.

Al parecer, estamos coexistiendo cada vez menos de forma biológica o negando aspectos de nuestra naturaleza humana, lo que nos aleja de nuestra propia realidad o intento de verdad, dejando de ser libres a estar controlados y automatizados.

Al ser humano, que habita en la complejidad social contemporánea, se le ha privado de la experiencia de su condición lúdica y su libre desarrollo. En la actualidad de las ideas y pensamientos que conforman su propia rutina y simultáneamente sus subjetivas bases culturales, se ha ido incapacitando de los dispositivos lúdicos que son parte sustancial de su naturaleza humana. Lo concreto es que la condición lúdica es una de las pocas dimensiones de las que el ser humano dispone de sí mismo, en cuanto a ser humano que Es y Está. La autonomía de existir libre, incierto, único, caótico, complejo. A sabiendas de que, en la cotidianeidad de los eventos convencionales abundan la ausencia de vértigo, el azar, improvisaciones, promiscuidades, aventuras, desventuras, lo extraordinario, nuevas experiencias compatibles y significantes. Paradójicamente, la vida está colmada de estos elementos. Además, a pesar de este control o la no existencia de espacios para potenciar nuestras interacciones lúdicas, éstas emergen de igual forma, no obstante, más limitadas, dañadas, marginadas al ocio (fugas).

Es tiempo de exhortar a los habitantes del gran mundo de la educación y de la escuela que co-construimos a hacerse la simple, pero no menos relevante disyuntiva de ¿Quedarse en estado de quietud permanente 0 fugarse urgentemente hacia lo lúdico?

\section{Referencias bibliográficas}

Agamben, G. (2011). Infancia e historia. Madrid: Edit. Adriana Hidalgo.

Buytendijk. (1935). El juego y su significado. Madrid: Revista de Occidente.

Caillois, R. (1986). Los Juegos y los Hombres: La máscara y el vértigo. México: Fondo de Cultura Económica.

Calvo, C. (2007). Del mapa escolar al territorio educativo. Diseñando la escuela desde la educación. Santiago: Edit. Nueva Mirada.

Huizinga, J. (2000). Homo Ludens. Madrid: Alianza editorial.

Illich, I. (1977). Educación sin escuelas. Barcelona: Editorial Península.

Luna, J. (2008). Complejidad en Educación. Santiago: Nau Llibres.

Maturana, H. (2003). Amor y juego: Fundamentos olvidados de lo humano 
desde el patriarcado a la democracia. Santiago: Edit. J.C. Sáez editor.

Naranjo, C. (2010). Cambiar la educación para cambiar el mundo. Santiago de Chile: Edit. Cuarto Propio.

Paymal, N. (2010). La Pedagogía 3000. La Paz, Bolivia: Edit. Librería Armonía y Ox La-Hun.

Schiller, F. (1990). Cartas sobre la educación estética del hombre. Barcelona: Anthropos. 